\title{
RE1 silencing transcription factor (REST) negatively regulates ALL1-fused from chromosome 1q (AF1q) gene transcription
}

\author{
Yuanyuan Hu' ${ }^{1}$ Qianwen Sun ${ }^{1}$, Chen Zhang ${ }^{1}$, Qingquan Sha ${ }^{1}$ and Xiulian Sun ${ }^{1,2^{*}}$
}

\begin{abstract}
Background: ALL1-fused from chromosome 1q (AF1q), originally considered as an oncogenic factor, has been implicated in neuronal development; however, its upstream regulatory mechanisms in neural system remained elusive.

Results: Our study showed that REST (RE1 silencing transcription factor), a key transcription factor in neurodevelopment, could down-regulate the gene expression of AF1q. The promoter assay identified a neuron-restrictive silencer element at -383 to -363 bp of human AF1q promoter. Electrophoretic mobility shift assay (EMSA) and chromatin immunoprecipitation (CHIP) confirmed the binding of REST to the NRSE in AF1a gene promoter. Additionally, the negative correlation between the expression levels of Af1 $q$ and Rest in mice neurodevelopment supported the negative regulation of AF1q by REST and the potential functions of AF1q in neurodevelopment.
\end{abstract}

Conclusion: These results demonstrate that REST regulates AF1 $q$ gene transcription through directly binding to a NRSE at -383 to -363 bp of AF1 q promoter.

Keywords: AF1q, REST, Promoter, Gene transcription, Neurodevelopment

\section{Background}

The $A F 1 q$ gene was initially identified as a mixed lineage leukaemia (MLL) fusion partner from an infant acute myelomonocytic leukemia patient carrying the $t(1 ; 11)$ (q21;q23) translocation [1], which encoded a highly conserved 90 amino acid residues protein containing a MLLT11 motif and a nuclear export signal [2] with no similarity to other known proteins [1]. An increase in AF1q mRNA levels had been shown in leukemic and immature hematopoietic cells [1]. MiR-29b can regulate AF1Q gene expression and lower expression of miR-29b was associated with poor overall survival of acute myeloid leukemia [3]. AF1q had been regarded as an oncogenic factor involved in thyroid oncocytic tumors, breast cancer and testicular germ cell tumors [4-8], though its function had not been well characterized. A recent study showed that AF1Q interacted with T cell factor 7 of Wnt

\footnotetext{
*Correspondence: xiulians@gmail.com

${ }^{2}$ Brain Research Institute, Qilu Hospital of Shandong University, 107

Wenhuaxi Rd., Jinan 250012, Shandong Province, China

Full list of author information is available at the end of the article
}

signaling pathway to regulate CD44 and promoted breast cancer metastasis [9]. In addition to the proposed oncogenic role, AF1q had been reported to play an important role in the development of neurons in the peripheral and central nervous systems [10]. The mouse Af1q, homologue of human $A F 1 q$, was found to be significantly up-regulated during the neuronal production from neural stem/progenitor cells [11]. AF1q was differentially expressed during neuronal differentiation [10], but the underlying regulatory mechanisms in neurodevelopment were unknown.

REST (RE1 silencing transcription factor) regulates embryonic and neural stem cells by affecting their derivatives [12-14] and participating in the self-renewal of neural stem cells [13] via regulating the transcription of target genes by binding to a 21-bp DNA element, which is called RE1-binding site/neuron-restrictive silencer element (RE1/NRSE) during neurodevelopment [15]. It was found that the expression of REST was decreased in cultured neurospheres derived from fetal Down syndrome (DS) brain [16] and in the brains of DS mouse models 
[17]. REST was decreased in Alzheimer's disease [10, 18]. REST, is expressed throughout early development [19] and acts as transcriptional silencer or activator, which is essential for the regulation of target genes during neuronal development [20, 21]. Downregulation of REST during neurogenesis is necessary for proper neuronal differentiation, while overexpression of REST in differentiating neurons interferes in neuronal gene expression and causes axon guidance errors [22]. REST modulated the expression of genes that were critical in normal neuronal functions including neurotransmitter receptors, synaptic proteins, and ion channels proteins [15, 16]. Recent studies have demonstrated that in contrast to the role of REST in the repression of Rest target genes in in vitro cultured neuronal cells, as well as in non-neuronal cells outside of the brain, the CoRest binding site of Rest is dispensable for embryonic neurogenesis in vivo [12].

In the present study, we demonstrated that $A F 1 q$ transcription could be down-regulated by REST. We also identified $A F 1 q$ promoter sequence and demonstrated that REST was a key transcriptional factor participating in AF1q down-regulation via a NRSE site at -383 to -363 bp of human $A F 1 q$ promoter. Furthermore, our study suggested that the expression of REST and AF1q were negatively correlated during neurodevelopment, implying that AF1Q may be involved in neurodevelopment.

\section{Results}

\section{REST inhibited human AF1q gene transcription}

To investigate whether $A F 1 q$ gene transcription was regulated by REST, the endogenous $A F 1 q$ mRNA levels were determined by RT-PCR in cells transfected with REST expression plasmid pREST or its pSuper based silencing vector psiREST. The knock-down efficiency of psiREST was detected by real time PCR using SYBR Green gene expression assay. Silencing plasmid pSuper was performed as control. Real time PCR confirmed that REST expression was reduced by psiREST to $37.57 \pm 3.81 \%$ of controls (Fig. 1a). REST mRNA level could be elevated or reduced by pREST or psiREST to $280.4 \pm 8.331$ and $56.81 \pm 4.813 \%(\mathrm{p}<0.05$; Fig. 1a). Similar results were obtained in the protein levels $(\mathrm{p}<0.05$; Fig. $1 \mathrm{~b})$. Overexpression of REST in HEK293 cells could significantly reduce $A F 1 q$ mRNA levels to $42.31 \pm 4.567 \%$ of controls $(\mathrm{p}<0.01$; Fig. 1c). Furthermore, knockdown of REST could markedly increase the $A F 1 q$ mRNA levels to $256.1 \pm 8.268 \%(\mathrm{p}<0.01$; Fig. $1 \mathrm{c})$. The data here indicated that REST was a negative regulator of $A F 1 q$ gene expression.

To further clarify the molecular mechanism of $A F 1 q$ gene transcription, we cloned 1811-bp fragment located in the $5^{\prime}$-flanking region of the human $A F 1 q$ gene
(Fig. 1d) into promoterless vector pGL3Basic. TSS represents the first base on exon 1 from Ensemble transcript ENST00000368921, which is 2802-bp upstream of translation start codon. Thus, the expression of luciferase activity in cells transfected with this plasmid depended on the insertion and proper orientation of a functional promoter upstream of the firefly luciferase gene. Plasmid pAF1q promoter $(-1349$ to $+462 \mathrm{bp})$ was transfected into HEK293 cells, and luciferase activity was measured by a luminometer to reflect $A F 1 q$ promoter activity. Plasmid pAF1q promoter transfected cells had significantly higher luciferase activity compared with controls $(0.9445 \pm 0.2401$ compared with $0.08008 \pm 0.006239$ relative luciferase units, $\mathrm{p}<0.05$; Fig. 1e, f), indicating that the $1.8-\mathrm{kb}$ fragment contains the functional promoter region of the human $A F 1 q$ gene.

To investigate whether $A F 1 q$ gene transcription could be regulated by REST, we cotransfected HEK293 cells with REST expression plasmid and $\mathrm{p} A F 1 q$ promoter, and examined the promoter activity of human $A F 1 q$ gene. A series of different mass ratio of REST and $A F 1 q$ promoter were selected and screened for modulatory effects on dual-luciferase assay. The transfected mass ratio of REST to $A F 1 q$ promoter was 1:3, 1:1, 3:1 and 5:1, respectively. The REST protein expression was determined by Western blotting (Fig. 1g). Dual-luciferase assay showed that REST overexpression decreased AF1q promoter activity and the effects depended on the mass ratio of REST to AF1q promoter. We could see that the AF1Q promoter activity was reduce by $19.34 \pm 8.499,43.59 \pm 6.122$, $53.24 \pm 6.251$, and $44.58 \pm 7.531 \%$ with increased expression of REST ( $<<0.05$; Fig. 1h). These results indicated that the transcription of human AF1q gene could be down regulated by REST.

\section{Identification of the functional NRSE site in AF1q gene promoter}

Above data showed that $A F 1 q$ gene promoter could be regulated by REST. Previous studied had shown that REST regulated the transcription of neural genes via binding to a NRSE site, a 21-bp consensus DNA sequence, which was present in the regulatory regions of neural genes. In this study, a computer-based transcription factor-binding site search by the JASPAR database revealed that the 1811-bp 5'-flanking region contained two putative NRSE located at -383 to -363 bp and +422 to +442 , which were the potential binding sites for REST. To identify the binding site, a series of luciferase reporter gene plasmids controlled by different upstream deletions of human $A F 1 q$ gene promoter region were constructed (Fig. 2a, b). Each deletion construct was transfected into HEK293 cells with REST expression plasmid, respectively. Dual-luciferase assay showed that 

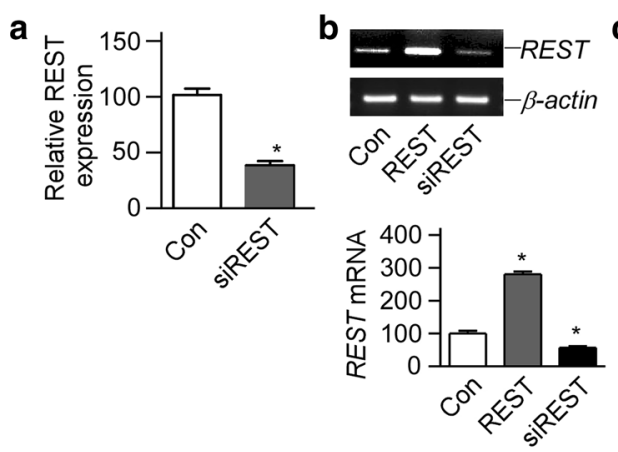
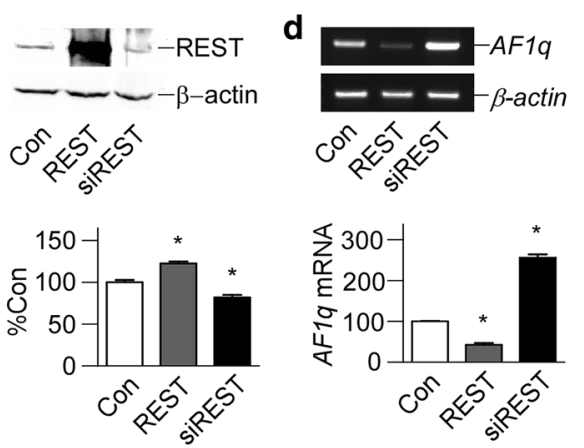

e

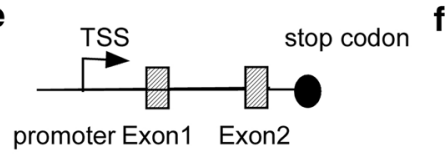

g

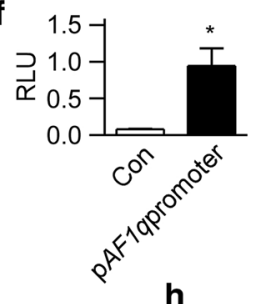

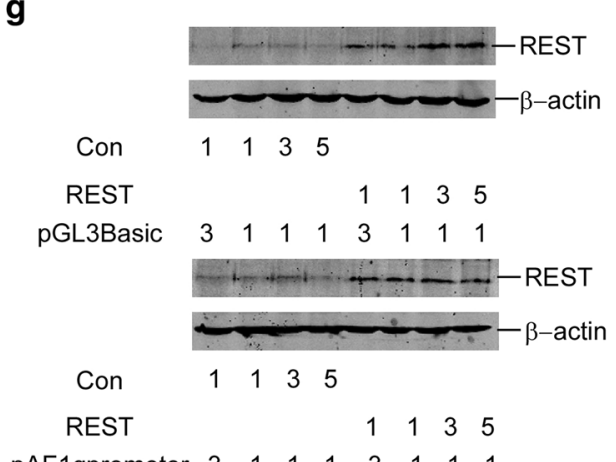

pAF1qpromoter $3 \begin{array}{lllllll}3 & 1 & 1 & 3 & 1 & 1 & 1\end{array}$

Fig. 1 REST inhibited human AF1q gene transcription. a Real time PCR showed that REST expression was decreased by psiREST. SYBR Green gene expression assay of REST was performed. 18S rRNA was used as the internal control. The values represent the mean \pm SE $(n=3)$. ${ }^{*} P<0.05$ by Student's $t$ test. Con control. b RT-PCR showed that REST expression construct pREST increased REST mRNA expression, and REST knock down construct psiREST decreased REST mRNA expression. $\beta$-actin was used as the internal control. The values represented the mean $\pm S E(n=3)$. ${ }^{*} P<0.01$ by Student's $t$ test. c The knockdown effect of psiREST was verified in protein level by Western blot. REST was detected with anti-REST antibody. $\beta$-actin was used as the internal control. The values represented the mean $\pm S E(n=3)$. ${ }^{*} P<0.01$ by Student's $t$ test. d RT-PCR showed that AF1q mRNA was repressed by REST overexpression in HEK293 cells. $\beta$-actin was used as the internal control. The values represented the mean $\pm S E(n=3)$. ${ }^{*} P<0.01$ by Student's $t$ test. e Genomic organization of AF1q gene. AF1q gene had two exons and one intron. TSS, transcription start site. f pAF1q-promoter contains the functional promoter of AF1a gene, containing the 1809-bp fragment from the human AF1a gene $5^{\prime} \mathrm{UTR}$, was transfected into HEK293 cells. +1 is the first base of the first exon. Dual-luciferase activity was measured by a luminometer $24 \mathrm{~h}$ after transfection. The values represented the mean $\pm S E(n=3) .{ }^{*} P<0.01$ by Student's $t$ test. $\mathbf{g}$ The expression of REST was examined by Western bolt. REST was detected with anti-REST antibody. Different mass ratios were marked under the Western blot figure. $\beta$-actin was used as the internal control. $\mathbf{h}$ AF1q gene promoter was inhibited by REST. REST was co-transfected with PAF1a promoter into HEK293 cells. Dual-luciferase activity was measured $24 \mathrm{~h}$ after transfection by a luminometer. The values represented the mean $\pm \operatorname{SE}(n=3) .{ }^{*} P<0.01$ by Student's $t$ test

REST significantly decreased $A F 1 q$ promoter activity of pAF1qAluc, but had no significant effect on pAF1qBluc (Fig. 2c), indicating that the region of -383 to $-363 \mathrm{bp}$ of $A F 1 q$ promoter might contain a NRSE. Moreover, the mutation of the NRSE site at -383 to -363 bp of pAF1qAluc abolished the effect of REST (Fig. 2e). These data indicated that the NRSE site located from -383 to -363 bp was essential for REST regulation.
To further confirm the NRSE located from -383 to $-363 \mathrm{bp}$ in $A F 1 q$ promoter was the binding site for REST, EMSA and ChIP were performed. A 21-bp doublestranded oligonucleotide probe containing the sequence of putative NRSE site in AF1q promoter was synthesized and end-labeled with IRDye 800 infrared dye. Consensus NRSE, mutant consensus NRSE, and the putative NRSE site in $A F 1 q$ promoter were also synthesized as cold probes. A shifted DNA-protein complex band was 
a
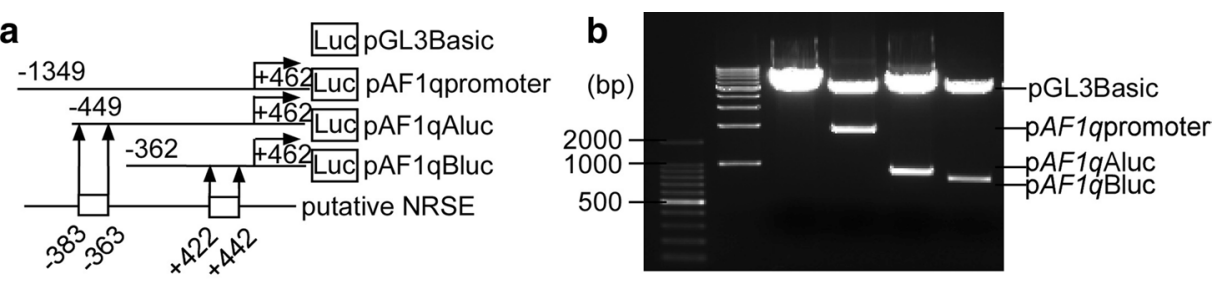

d

The NRSE site at -383 to $-363 \mathrm{bp}$ of AF1q promoter

AF1qNRSE: TTAGCTGGGCGTGGTGGCGGA AF1qNRSEmut: TTAGCTGGaagTctgGaagGA

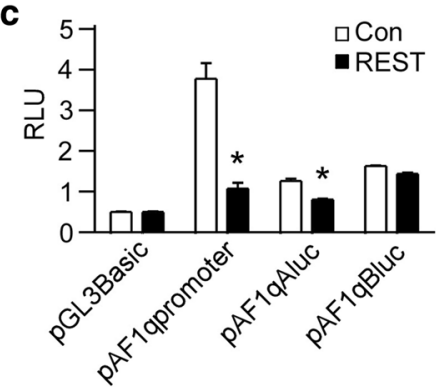

e

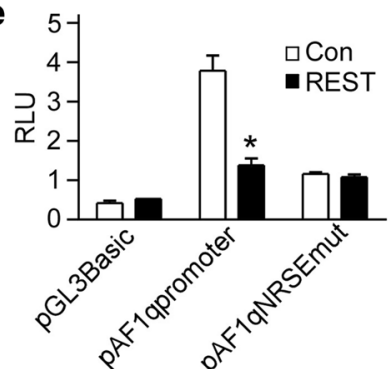

Fig. 2 Identification of the functional NRSE site in AF1q gene promoter. a Schematic diagrams of the AF1q promoter deletion constructs consisting of a 5'-flanking region with serial deletions cloned into the promoter-less vector plasmid $\mathrm{pGL3-Basic} \mathrm{in} \mathrm{front} \mathrm{of} \mathrm{the} \mathrm{luciferase} \mathrm{reporter} \mathrm{gene.} \mathrm{Arrow}$ indicated the direction of transcription. The numbers represented the end points of each construct. The positions of two putative NRSE sites were shown at the bottom. b The deletion plasmids were confirmed by sequencing and restriction enzyme digestion on a $1.5 \%$ agarose gel. Vector size is $4.7 \mathrm{~kb}$, and the AF1q gene 5'-flanking fragment inserts ranged from 0.6 to $1.8 \mathrm{~kb}$. c HEK293 cells were co-transfected with REST expression vector and various AF1 $q$ promoter deletion constructs. Plasmid pRL-TK was used to normalize the transfection efficiency, and luciferase activity was measured at $24 \mathrm{~h}$ by a luminometer. The values represented the mean $\pm \mathrm{SE}(\mathrm{n}=3)$. ${ }^{*} P<0.01$ by Student's $t$ test. $\mathbf{d}$ pAF1qNRSEmut was made to contain the mutant NRSE site at -383 to -363 of $\mathrm{p}$ AF1 qAluc, where (5'-TTAGCTGGGCGTGGTGGCGGA-3') was mutated to (5'-TTAGCTGGaaGTctgGaaGGA-3'). e HEK293 cells were co-transfected with REST expression vector and AF1q promoter or pAF1qNRSEmut. Plasmid pRL-TK was used to normalize the transfection efficiency, and luciferase activity was measured at $24 \mathrm{~h}$ by a luminometer. The values represented the mean $\pm S E(n=3)$. ${ }^{*} P<0.01$ by Student's $t$ test

detected after incubation of the labeled consensus NRSE probe with HeLa cell nuclear extract (lane 2 of Fig. 3a). The binding intensity of the shifted band was significantly reduced by applying 100 - or 200 -fold molar excess of unlabeled consensus NRSE competitive oligonucleotides (lane 4 and 5 of Fig. 3a). The incubation of 200 -fold excessive mutant NRSE oligonucleotides had no competitive effect in the shifted NRSE-REST band (lane 6 and 7 of Fig. 3a). The addition of 100- and 200-fold excess of NRSE-AF1q, corresponding to the NRSE site from -383 to $-363 \mathrm{bp}$ of the $A F 1 q$ promoter region, reduced the NRSE-REST shifted band in a dosage-dependent manner (lane 8 and 9 of Fig. 3a). The supershift band with the addition of anti-REST antibody further confirmed the specific binding between REST and AF1Q-NRSE site (Fig. 3b). These results further demonstrated that the NRSE-AF1q site at -383 to -363 in human $A F 1 q$ promoter was the binding site of REST.

To confirm that the NRSE-AF1q site verified in vitro can actually bind to REST in vivo, ChIP was employed to pulldown the REST bound DNA. The anti-REST antibody actually immunoprecipitated REST protein as showed in
Fig. 3c. Anti-RNA Polymerase II IP was used as positive control and IgG IP was used as negative control for ChIP. Purified DNA was then analyzed by PCR using Control Primers specific for the GAPDH promoter (Fig. 3d). PCR product was observed in the anti-RNA Polymerase II ChIP (lane 2, Fig. 3d), but not in the IgG ChIP (lane 3, Fig. 3d). ChIP-PCR results showed that REST antibody effectively immunoprecipitated the AF1q-NRSE site (lane 2, Fig. 3e). And the ChIP band was greatly reduced in cells with REST knocked down (lane 5, Fig. 3e). AF1q-NRSE PCR product was also observed in the anti-RNA Polymerase II ChIP (lane 6, Fig. 3e) and not in the IgG ChIP (lane 3, Fig. 3e). AF1q promoter specific DNA was also observed in the Input (lane 1, Fig. 3e). Taken together, these data indicated that the NRSE site, corresponding to $A F 1 q$ promoter -383 to $-363 \mathrm{bp}$, was responsible for the down-regulation of $A F 1 q$ gene by REST.

\section{Af1q expression was negatively correlated with Rest in mice neurodevelopment}

To investigate whether there are interactions between $A f 1 q$ and Rest during neurodevelopment, we detected 


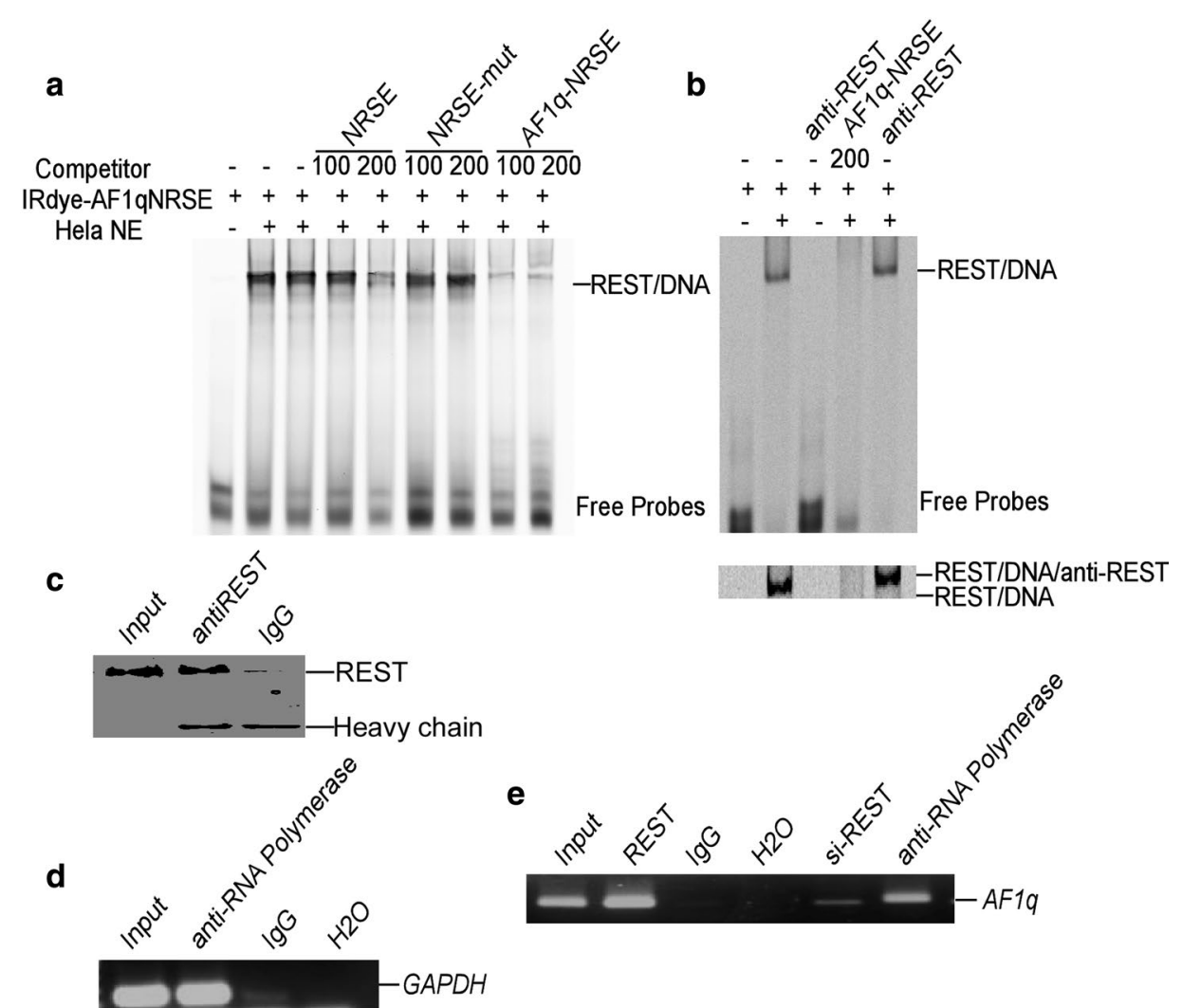

Fig. 3 a EMSA was performed with IRDye 800-labeled NRSE site of AF1q promoter. Competition assays were performed by further adding different concentration of molar excess of unlabeled competitive oligonucleotides. b The addition of anti-REST antibody further shifted DNA-protein complex band. Lower panel showed a longer running time. c The anti-REST antibody actually immunoprecipitated REST protein. Lane 1 was input. Lane 2 was immunoprecipitates by anti-REST antibody. Lane 3 was immunoprecipitates by lgG antibody. And the lower bands were antibody heavy chain. d Anti-RNA polymerase II antibody was used to immunoprecipitate the GAPDH promoter region in CHIP assay in HEK293 cells. A pair of primers targeting GAPDH was used to amplify GAPDH. Signals amplified from input were used as size markers for PCR. IgG and $\mathrm{H}_{2} \mathrm{O}$ were used as negative controls. e Anti-REST antibody was used to immunoprecipitate the cross-linked REST-DNA complex in CHIP assay in HEK293 cells. A pair of primers targeting AF1q was used to amplify AF1q-REST. Signals amplified from input were used as size markers for PCR. IgG and $\mathrm{H}_{2} \mathrm{O}$ were used as negative controls. And anti-RNA Polymerase II antibody was used as positive control. Si-REST was from cells transfected with psiREST

mRNA levels of $A f 1 q$ and Rest in the developing mice brains. RNA was extracted from normal mouse brains aging at embryonic days 13.5 and 18 and postnatal day 1 (P1), P7, P14 and adult. The real-time RT-PCR results showed that mRNA levels of Af1q and Rest were coordinately expressed with significant negative correlation during neurodevelopment $(\mathrm{p}=0.0010, \mathrm{r}=-0.8182$ by Spearman correlation; Fig. 4a). The plot showed that the E18 measured point was deviated from the statistically supported tendency. These data further demonstrated that $A f 1 q$ gene expression was negatively regulated by Rest in neurodevelopment.

\section{Discussion}

Our study here showed that REST, a key transcription factor in neurodevelopment, can down-regulate the gene

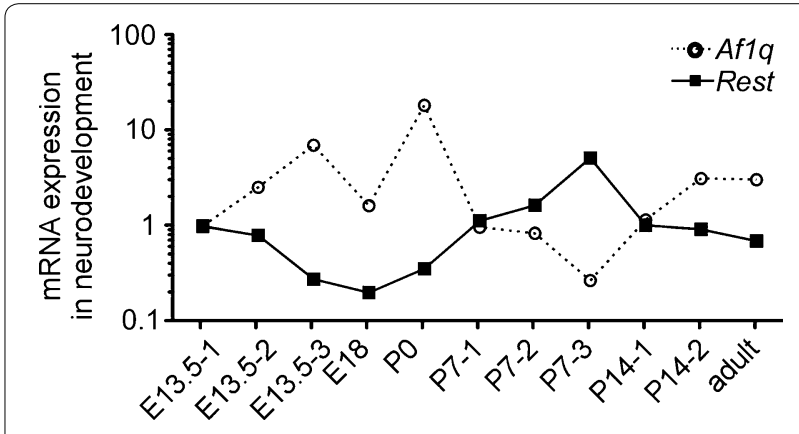

Fig. 4 Af1q was negatively correlated with REST in mice neurodevelopment. a Quantitative RT-PCR was performed on CDNA templates prepared from normal mouse brain aging at embryonic days 13.5 (E13.5) and 18.5 (E18.5); at postnatal P1, P7, and P14; and in adult mice. One to three mice were used in each time point as indicated by the numbers after the hyphens. $P<0.05$ by Spearman correlation test 
expression of $A F 1 q$ through directly binding to a NRSE at -383 to -363 bp of $A F 1 q$ promoter. AF1q, originally considered as an oncogenic factor, is highly expressed in normal hematopoietic tissues, leukemic cell lines and neuronal cells in central nervous system $[1,10,11,23]$. We can see that the promoter activity in pAF1q promoter is higher than the promoter activity in pAF1qAluc, indicating the region of -1349 to -449 bp containing binding sites for some activators. The decrease of inhibitory effect by REST on pAF1QAluc suggested that the region of -1349 to -449 bp contained binding sites for REST cofactors.

Our study here elucidated the regulatory mechanism of $A F 1 q$ by REST. REST, acting as transcriptional silencer or activator, was essential for the regulation of target genes during neuronal development $[20,21]$. REST is required to repress the expression of neuronal genes in undifferentiated neuronal tissue. Expression of REST was highest in embryonic stem cells, but it was decreased while ESCs were differentiated into neuronal stem cells, and it was at low level in mature adult neuronal cells [20]. In addition to participate in neurogenesis, Rest also mediated the interactions between neuron and glia, which was associated with synaptic and neuronal network plasticity and homeostasis in the brain $[24,25]$. All these indicated that REST is a key transcription factor in neurogenesis. As a target gene of REST, how AF1q functions in neurogenesis remains elusive. It will be interesting to examine the function of AF1q in neural stem cells and neuronal differentiation. It was reported that REST expression is a protective factor in aging and is decreased in neurodegenerative diseases such as Alzheimer's disease [18]. It will be interesting to check the expression level of AF1q in some neurodegenerative diseases such as Alzheimer's disease.

Overexpression of REST has been found in human medulloblastomas, glioblastoma and neuroblastomas [26], in which REST acted as an oncogene to maintain the stem character of neural cells [27]. REST can also act as a tumor suppressor in carcinomas including lung, breast and colon [27]. Though AF1q is regard as an oncogene, the expression level of AF1q is unknown in these cancers. It will be interesting to examine the expression of AF1Q in these cancers associated with REST dysfunction.

\section{Conclusions}

In summary, the current study provides a molecular model for REST in negative regulation of $A F 1 q$ promoter activity and mRNA expression. These results will help to better understand the role of $A F 1 q$ gene in in neural stem cells and neuronal differentiation.

\section{Methods}

\section{Plasmids construction}

The $5^{\prime}$-upstream region of human $A F 1 q$ gene $(-1349$ to +462 bp, pAF1q-promoter) was obtained by PCR of genomic DNA isolated from BAC-human-rp11 using a pair of primers (5'-CGGCTAGCAGGTCTCCACC CTGTCCCTGC- $3^{\prime}$ and $5^{\prime}$-CCCTCGAGTTCCCTCCAC CCAGCTCTGGTC-3'). The first base of the first exon is referred as $b p+1$. Then they were cloned into pGL3 basic vector (Promega, Madison, WI) containing firefly luciferase reporter gene. Primers used to generate a series of promoter deletion plasmids were as follows: forward primers, 5'-CGGCTAGCGTCAGGAGTTCCAG ACCAGC-3' (pAF1qAluc), 5'-CGGCTAGCTGTAATCC CAGCTACTTGGG-3' (pAF1qBluc), and reverse primers: 5'-CCCTCGAGCAGAAATGGCCTTGTTCTCT-3'. The $\mathrm{p} A F 1 q \mathrm{NRSEmut}$ was constructed from pAF1QAluc in which the NRSE site $5^{\prime}$-TTAGCTGGGCGTGGTGG CGGA-3' was mutated to $5^{\prime}$-TTAGCTGGaaGTctgGaa GGA- ${ }^{\prime}$. All the constructs were verified by sequencing and restriction enzyme digestions. Human REST siRNA was generated using pSuper vector. The target sequence for human REST siRNA is GCTACAATACTAATCGATA. The two strands of RESTsuper sense (5'-gatcccGCTACAATACTAATCG ATAttcaagagaTATCGATTAGTATTGTAGCttttta) and RESTsuper antisense (5'-agcttaaaaaGCTACAATACTA ATCGATAtctcttgaaTATCGATTAGTATTGTAGCggg) were annealed and inserted into pSuper plasmid to generate the pSuper-REST construct. The vectors pREST were generated as previously described [28].

\section{Cell culture, transfection and dual-luciferase assay}

HEK293 cells were cultured as previously described [29]. All cells were maintained at $37^{\circ} \mathrm{C}$ in an incubator containing $5 \% \mathrm{CO}_{2}$. All transfections were carried out with lipofectamine ${ }^{\mathrm{TM}} 2000$ transfection reagent (Invitrogen) according to the manufacturer's instructions. Luciferase activity was determined as previously described [21].

\section{RT-PCR}

Total RNA was isolated from mouse brains or cells using TRIzol reagent (Invitrogen). The mRNA of REST and $A F 1 q$ was quantified by TOYOBO R SYBR Green gene Expression Analysis kit (TOYOBO, Japan). Primers for real-time quantitative and semi-quantitative PCR were as follows: human $A F 1 q$ (270 bp), forward, 5'-CCG CTCGAGGCCACCATGAGGGACCCTGTGAG-3', and reverse, 5'-GGGGTACCGAGCAAGTCCAGTTCGAA G-3'; human REST (137 bp), forward, 5'-ACTCATCA CGGAGAACGCCC-3' ${ }^{\prime}$, and reverse, 5'-GAGGCCACA TAACTGCACTG-3'; mouse Rest (244 bp), forward, 
5'-CGAGTCTCAGGAAATTGATGA-3', and reverse, $5^{\prime}$-GCCGTTACCCACTCACTAATAC-3'; human and mouse $\beta$-actin (141 bp), forward, $5^{\prime}$-GACAGGATGCAG AAGGAGAT-3', and reverse, 5'-TGATCCACATCTG CTGGAAGGT- $3^{\prime}$. All animal protocols were approved by Shandong University Institutional Animal Care and Use Committee and by the Institutional Ethics Committee on Animal Research of Qilu Hospital.

\section{EMSA and ChIP}

The sense sequences of AF1qNRSE, consensus NRSE and mutant AF1qNRSE were 5'-AAAGATTAGCTGG GCGTGGTGGCGGATGCCTGTA, 5'-TTCAGCACCA CGGACAGCGCC, 5'-AAAGATTAGCTGGAAGTCTG GAAGGATGCCTGTA. EMSA and ChIP were performed as previously described [28]. Chip-PCR was performed using the DNA reversed from the cross-linked complex with a pair of primers (NRSE-AF1q:5'-GCCT CCGGTTGTACCACT- ${ }^{\prime}$ and 5'-AGCGATTCTCCTGC CTCA-3'). Control primer specific for human GAPDH were $5^{\prime}$-TACTAGCGGTTTTACGGGCG-3' ${ }^{\prime}$ and $5^{\prime}$-TC GAACAGGAGGAGCAGAGAGCGA-3'.

\section{Western blot analysis}

Western blot was performed as previously described [21]. Anti-REST antibody was from Millipore (\#DAM15).

\section{Data analysis}

All experiments were repeated three to five times. In figures one representative picture was shown; quantifications were from three or five independent experiments. The values represent the mean \pm SEM. The data were evaluated for statistical significance with Student's $t$ test analysis.

\section{Authors' contributions}

$\mathrm{YH}$ participated in drafting of the manuscript and carried out the promoter assays, EMSA and ChIP assays. QS, CZ and QS helped YH in promoter construct cloning and bioinformatic analysis. XS designed the experiment and wrote the manuscript. All authors read and approved the final manuscript.

\section{Author details}

1 Department of Neurology, Qilu Hospital Shandong University, 107 Wenhuaxi Rd., Jinan 250012, Shandong Province, China. ${ }^{2}$ Brain Research Institute, Qilu Hospital of Shandong University, 107 Wenhuaxi Rd., Jinan 250012, Shandong Province, China.

\section{Acknowledgements}

This work was supported by National Natural Science Foundation of China Grant 81171030.

\section{Compliance with ethical guidelines}

\section{Competing interests}

The authors declares that they have no competing interests.

Received: 2 March 2015 Accepted: 27 August 2015

Published online: 05 September 2015

\section{References}

1. Tse W, Zhu W, Chen HS, Cohen A. A novel gene, AF1q, fused to MLL in $\mathrm{t}(1 ; 11)$ (q21;q23), is specifically expressed in leukemic and immature hematopoietic cells. Blood. 1995;85(3):650-6.

2. Parcelier A, Maharzi N, Delord M, Robledo-Sarmiento M, Nelson E, Belakhdar-Mekid H, Pla M, Kuranda K, Parietti V, Goodhardt M, et al. AF1q/ MLLT11 regulates the emergence of human prothymocytes through cooperative interaction with the Notch signaling pathway. Blood. 2011;118(7):1784-96.

3. Xiong Y, Li Z, Ji M, Tan AC, Bemis J, Tse JV, Huang G, Park J, Ji C, Chen J, et al. MIR29B regulates expression of MLLT11 (AF1Q), an MLL fusion partner, and low MIR29B expression associates with adverse cytogenetics and poor overall survival in AML. Br J Haematol. 2011;153(6):753-7.

4. Co NN, Tsang WP, Wong TW, Cheung HH, Tsang TY, Kong SK, Kwok TT. Oncogene AF1q enhances doxorubicin-induced apoptosis through BAD-mediated mitochondrial apoptotic pathway. Mol Cancer Ther. 2008;7(10):3160-8.

5. Jacques C, Baris O, Prunier-Mirebeau D, Savagner F, Rodien P, Rohmer V, Franc B, Guyetant S, Malthiery Y, Reynier P. Two-step differential expression analysis reveals a new set of genes involved in thyroid oncocytic tumors. J Clin Endocrinol Metabol. 2005;90(4):2314-20.

6. Li DQ, Hou YF, Wu J, Chen Y, Lu JS, Di GH, Ou ZL, Shen ZZ, Ding J, Shao $\mathrm{ZM}$. Gene expression profile analysis of an isogenic tumour metastasis model reveals a functional role for oncogene AF1Q in breast cancer metastasis. Eur J Cancer. 2006;42(18):3274-86.

7. Skotheim Rl, Autio R, Lind GE, Kraggerud SM, Andrews PW, Monni O, Kallioniemi O, Lothe RA. Novel genomic aberrations in testicular germ cell tumors by array-CGH, and associated gene expression changes. Cell Oncol Off J Int Soc Cell Oncol. 2006;28(5-6):315-26.

8. Chang XZ, Li DQ, Hou YF, Wu J, Lu JS, Di GH, Jin W, Ou ZL, Shen ZZ, Shao ZM. Identification of the functional role of AF1Q in the progression of breast cancer. Breast Cancer Res Treat. 2008;111(1):65-78.

9. Park J, Schlederer M, Schreiber M, Ice R, Merkel O, Bilban M, Hofbauer S, Kim S, Addison J, Zou J et al. AF1q is a novel TCF7 co-factor which activates CD44 and promotes breast cancer metastasis. Oncotarget. 2015.

10. Yamada M, Clark J, lulianella A. MLLT1 1/AF1q is differentially expressed in maturing neurons during development. Gene Expr Patterns GEP. 2014;15(2):80-7.

11. Lin HJ, Shaffer KM, Sun Z, Jay G, He WW, Ma W. AF1q, a differentially expressed gene during neuronal differentiation, transforms HEK cells into neuron-like cells. Brain Res Mol Brain Res. 2004;131(1-2):126-30.

12. Aoki H, Hara A, Era T, Kunisada T, Yamada Y. Genetic ablation of Rest leads to in vitro-specific derepression of neuronal genes during neurogenesis. Development. 2012;139(4):667-77.

13. Gao Z, Ure K, Ding P, Nashaat M, Yuan L, Ma J, Hammer RE, Hsieh J. The master negative regulator REST/NRSF controls adult neurogenesis by restraining the neurogenic program in quiescent stem cells. J Neurosci Off J Soc Neurosci. 2011;31(26):9772-86.

14. Sun YM, Cooper M, Finch S, Lin HH, Chen ZF, Williams BP, Buckley NJ. Restmediated regulation of extracellular matrix is crucial for neural development. PLoS One. 2008;3(11):e3656

15. Chong JA, Tapia-Ramirez J, Kim S, Toledo-Aral JJ, Zheng Y, Boutros MC, Altshuller YM, Frohman MA, Kraner SD, Mandel G. REST: a mammalian silencer protein that restricts sodium channel gene expression to neurons. Cell. 1995;80(6):949-57.

16. Bahn S, Mimmack M, Ryan M, Caldwell MA, Jauniaux E, Starkey M, Svendsen $C N$, Emson P. Neuronal target genes of the neuron-restrictive silencer factor in neurospheres derived from fetuses with Down's syndrome: a gene expression study. Lancet. 2002;359(9303):310-5.

17. Canzonetta C, Mulligan C, Deutsch S, Ruf S, O'Doherty A, Lyle R, Borel C, Lin-Marq N, Delom F, Groet J, et al. DYRK1A-dosage imbalance perturbs NRSF/REST levels, deregulating pluripotency and embryonic stem cell fate in Down syndrome. Am J Hum Genet. 2008;83(3):388-400.

18. Lu T, Aron L, Zullo J, Pan Y, Kim H, Chen Y, Yang TH, Kim HM, Drake D, Liu $X S$, et al. REST and stress resistance in ageing and Alzheimer's disease. Nature. 2014;507(7493):448-54.

19. Ballas N, Battaglioli E, Atouf F, Andres ME, Chenoweth J, Anderson ME, Burger C, Moniwa M, Davie JR, Bowers WJ, et al. Regulation of neuronal traits by a novel transcriptional complex. Neuron. 2001;31(3):353-65. 
20. Ballas N, Grunseich C, Lu DD, Speh JC, Mandel G. REST and its corepressors mediate plasticity of neuronal gene chromatin throughout neurogenesis. Cell. 2005;121(4):645-57.

21. Sun YM, Greenway DJ, Johnson R, Street M, Belyaev ND, Deuchars J, Bee T, Wilde S, Buckley NJ. Distinct profiles of REST interactions with its target genes at different stages of neuronal development. Mol Biol Cell. 2005;16(12):5630-8

22. Juhila J, Sipila T, Icay K, Nicorici D, Ellonen P, Kallio A, Korpelainen E, Greco D, Hovatta I. MicroRNA expression profiling reveals miRNA families regulating specific biological pathways in mouse frontal cortex and hippocampus. PLoS One. 2011;6(6):e21495.

23. Busson-Le Coniat M, Salomon-Nguyen F, Hillion J, Bernard OA, Berger R. MLL-AF1q fusion resulting from $\mathrm{t}(1 ; 11)$ in acute leukemia. Leukemia. 1999;13(2):302-6.

24. Abrajano JJ, Qureshi IA, Gokhan S, Zheng D, Bergman A, Mehler MF. Differential deployment of REST and COREST promotes glial subtype specification and oligodendrocyte lineage maturation. PLoS One. 2009;4(11):e7665.
25. Kohyama J, Sanosaka T, Tokunaga A, Takatsuka E, Tsujimura K, Okano H, Nakashima K. BMP-induced REST regulates the establishment and maintenance of astrocytic identity. J Cell Biol. 2010;189(1):159-70.

26. Lawinger P, Venugopal R, Guo ZS, Immaneni A, Sengupta D, Lu W, Rastelli L, Marin Dias Carneiro A, Levin V, Fuller GN, et al. The neuronal repressor REST/NRSF is an essential regulator in medulloblastoma cells. Nature Med. 2000;6(7):826-31.

27. Negrini S, Prada I, D'Alessandro R, Meldolesi J. REST: an oncogene or a tumor suppressor? Trends Cell Biol. 2013;23(6):289-95.

28. Lu M, Zheng L, Han B, Wang L, Wang P, Liu H, Sun X. REST regulates DYRK1A transcription in a negative feedback loop. J Biol Chem. 2011;286(12):10755-63.

29. Sun X, He G, Qing H, Zhou W, Dobie F, Cai F, Staufenbiel M, Huang $L E$, Song W. Hypoxia facilitates Alzheimer's disease pathogenesis by up-regulating BACE1 gene expression. Proc Natl Acad Sci USA. 2006;103(49):18727-32.

\section{Submit your next manuscript to BioMed Central and take full advantage of:}

- Convenient online submission

- Thorough peer review

- No space constraints or color figure charges

- Immediate publication on acceptance

- Inclusion in PubMed, CAS, Scopus and Google Scholar

- Research which is freely available for redistribution

Submit your manuscript at

www.biomedcentral.com/submit

C Biomed Central 